\title{
Asynchronous Group Mutual Exclusion in Ring Networks (Extended Abstract)
}

\author{
Kuen-Pin $\mathrm{Wu}$ \\ Department of Electrical Engineering \\ National Taiwan University \\ Taipei, Taiwan \\ kpw@orchid.ee.ntu.edu.tw
}

\author{
Yuh-Jzer Joung* \\ Department of Information Management \\ National Taiwan University \\ Taipei, Taiwan \\ joung@ccms.ntu.edu.tw
}

\begin{abstract}
The design issues for group mutual exclusion have been modeled by Joung as the Congenial Talking Philosophers, and solutions for shared-memory models and complete message-passing networks have been proposed [2, 3]. These solutions, however, cannot be straightforwardly and efficiently converted to ring networks where each philosopher can only communicate directly with its two neighboring philosophers. As rings are also a popular network topology, in this paper we focus the Congenial Talking Philosophers on ring networks and present an efficient and highly concurrent distributed algorithm for the problem.
\end{abstract}

\section{Introduction}

The design issues for mutual exclusion between groups of processes have recently been modeled by Joung [2] as the Congenial Talking Philosophers, which concerns a set of $N$ philosophers $p_{1}, p_{2}, \ldots, p_{N}$ which spend their time thinking alone and talking in a forum. Initially, all philosophers are thinking. From time to time, when a philosopher is tired of thinking, it wishes to attend a forum of its choice. Given that there is only one meeting room, a philosopher attempting to enter the meeting room to attend a forum can succeed only if the meeting room is empty (and in this case the philosopher starts the forum), or some philosopher interested in the same forum is already in the meeting room (and in this case the philosopher joins this ongoing forum). We assume that when a philosopher has attended a forum, it spends an unpredictable but finite amount of time in the forum. After a philosopher leaves a forum (and exits the meeting room) $)^{1}$, it returns to thinking. The problem is to design an algorithm for the philosophers satisfying the following requirements:

mutual exclusion: if some philosopher is in a forum, then no other philosopher can be in a different forum

${ }^{*}$ This research was supported in part by the National Science Council, Taipei, Taiwan, under Grants NSC 85-2213-E-002-059 and NSC 86-2213E-002-053.

${ }^{1}$ Throughout the paper, "in a forum" is synonymously with "in the meeting room." So, "to attend/leave a forum" is synonymously with "to enter/exit the meeting room." simultaneously.

bounded delay: a philosopher attempting to attend a forum will eventually succeed.

concurrent entering: if some philosophers are interested in a forum and no philosopher is interested in a different forum, then the philosophers can attend the forum concurrently.

Solutions for the Congenial Talking Philosophers problem for shared-memory models and complete messagepassing networks have been proposed $[2,3]$. These solutions, however, cannot be straightforwardly and efficiently converted to ring networks where each philosopher can only communicate directly with its two neighboring philosophers. As rings are also a popular network topology, in this paper we focus the problem on ring networks where the $N$ philosophers $p_{0}, p_{1}, \ldots, p_{N-1}$ are connected via a ring network so that each philosopher $p_{i}$ can only send messages directly to its immediate successor $p_{i+1}$. (Unless stated, otherwise, additions and subtractions on indices of philosophers are to be interpreted modulo $N$.) We shall assume that philosophers are distinguished by their unique IDs, and that every message will eventually be delivered to its destination. However, we do not require messages to be delivered in the order sent.

As we shall see, some solutions for the Congenial Talking Philosophers problem may be easy to design. However, their "performance" in practical applications may be unsatisfactory. So Joung has also proposed four measurements to evaluate the algorithms. We shall briefly discuss these measurements in the following section. For more details, please refer to $[2,3]$. Section 3 presents a straightforward solution to the Congenial Talking Philosophers problem. The solution easily satisfies the three problem requirements, and has complexity measures that "appear" to be acceptable. However, when putting the algorithm in simulation, its weakness becomes evident: the performance is only slightly better than imposing strict mutual exclusion on every entry to the meeting room. A more concurrent solution is therefore presented in Section 4. Section 5 concludes.

\section{Complexity Measures}

Solutions for the Congenial Talking Philosophers problem can be evaluated from the following four perspectives: 
messages, time, context-switch, and degree of concurrency.

Message complexity is concerned with how many messages the system generates per entry to the critical sectionthe meeting room.

For time, one is concerned with how long a philosopher $p_{i}$ should wait before it enters the critical section from the time it has made a request (by sending out messages to other philosophers). More formally, define a passage through $\mathrm{X}$ by $p_{i}$ to be an interval $\left[t_{1}, t_{2}\right]$, where $t_{1}$ is the time $p_{i}$ enters the meeting room to attend $\mathrm{X}$, and $t_{2}$ is the time it exits the meeting room. The attribute of the passage is denoted by $\left\langle p_{i}, \mathrm{X}\right\rangle$. When no confusion is possible, intervals and attributes are used interchangeably to represent passages. We often say that the passage is initiated at $t_{1}$, and is completed at $t_{2}$. The passage is ongoing at any time in between $t_{1}$ and $t_{2}$.

Let $T$ be the set of passages that may be initiated after a philosopher $p_{i}$ has made a request for a forum, and that must be completed before $p_{i}$ can enter the meeting room. The time complexity is measured by $\Omega(T)$, where $\Omega(T)$ is the size of a minimal set $R \subset T$ such that for every passage $\alpha \in T$, every time instance in $\alpha$ is contained in some $\beta \in R$.

The context-switch complexity is measured by the maximum number of rounds of passages that may be initiated after a philosopher has made a request for $\mathrm{X}$, but before a round of $\mathrm{X}$ is initiated in which $p_{i}$ can make a passage through $\mathrm{X}$. A round of passages through $\mathrm{Y}$ (or simply a round of $\mathrm{Y}$ ) is a maximal set of consecutive passages through forum $Y$.

The (maximum) degree of concurrency is defined by the maximum number of entries to the meeting room that can still be made while some philosopher is in the meeting room and another philosopher is waiting for a different forum.

Note that the above complexity measures all concern worst-case scenarios. Due to the dynamic nature of the problem, an average-case analysis is extremely complicated and so simulation studies [5] are also suggested by Joung [3].

\section{A Straightforward Solution}

We first present a straightforward solution to the Congenial Talking Philosophers problem over a ring network. In the algorithm, which we shall refer to as CTP-Ring1 (where CTP stands for Congenial Talking Philosophers), each philosopher is in one of the following three states: thinking, meaning that it is not interested in any forum; waiting, meaning that it is waiting for a forum; and talking, meaning that it is in a forum. Moreover, each philosopher maintains a variable $S N$ recording the maximum sequence number seen by the philosopher. The variable will be maintained similar to Lamport's logical clock [4]. That is, each philosopher initializes its $S N$ to 0 and, whenever it learns of a sequence number larger than its own $S N$, it advances its $S N$ to that number. Furthermore, a philosopher increments its $S N$ by 1 when it wishes to attend a forum.

Initially, every philosopher is in state thinking. When a philosopher $p_{i}$ wishes to attend a forum $\mathrm{X}$, it enters state waiting, increments its $S N$ by 1 , and sends a request message $\operatorname{Req}(\langle i, s n\rangle, \mathrm{X})$ to its successor $p_{i+1}$, where $s n$ is the new value of $p_{i}$ 's $S N$. Philosopher $p_{i}$ remains in state waiting until its request $\operatorname{Req}(\langle i, s n\rangle, \mathrm{X})$ is returned. Then, $p_{i}$ enters the meeting room to attend $\mathrm{X}$. After finishing the forum, $p_{i}$ returns to state thinking.

When $p_{i+1}$ receives a request message $\operatorname{Req}\left(\left\langle j, s n_{j}\right\rangle, \mathrm{X}\right)$ from $p_{i}$, it either forwards the request to its successor $p_{i+2}$, or detains the request in its message queue; the decision depends on $p_{i+1}$ 's state. $p_{i+1}$ forwards the request if one of the following conditions is satisfied: (1) it is in state thinking, (2) it is also interested in X, or (3) it is interested in a different forum and has a priority lower than $p_{j}$. A philosopher's priority is assigned as follows: when the philosopher has sent out a request $\operatorname{Req}\left(\left\langle j, s n_{j}\right\rangle, \mathrm{X}\right)$, it obtains a priority $\left\langle j, s n_{j}\right\rangle$, and it possesses the priority until it has left X. A priority $\left\langle j, s n_{j}\right\rangle$ is higher than $\left\langle k, s n_{k}\right\rangle$, denoted by $\left\langle j, s n_{j}\right\rangle>\left\langle k, s n_{k}\right\rangle$ if, and only if, $s n_{j}<s n_{k}$, or $s n_{j}=s n_{k}$ and $j<k$. For simplicity, we assume that a philosopher's priority is set to a minimal value $\langle i, \infty\rangle$ when it is not interested in any forum; that is, when it is in state thinking.

On the other hand, $p_{i+1}$ detains the request if none of the above three conditions is satisfied. In other words, $p_{i+1}$ detains the request if it is interested in a different forum $Y$ and has a priority higher than $\left\langle j, s n_{j}\right\rangle . p_{i+1}$ detains the request until it has finished $Y$. Then, $p_{i+1}$ forwards the request (and all other requests it has held) to its successor $p_{i+2}$.

It can be seen that the algorithm satisfies all three requirements of the Congenial Talking Philosophers problem. For mutual exclusion, observe that by the way sequence numbers are maintained, if a philosopher $p_{j}$ requests a forum after it has received a request $\operatorname{Req}(\langle i, s n\rangle, \mathrm{X})$ issued by $p_{i}$, then $p_{j}$ must obtain a priority for its request lower than $\langle i, s n\rangle$. So, when $p_{j}$ 's request circles to $p_{i}$, if the request is for a forum different from $X$, then the request must be detained by $p_{i}$ until $p_{i}$ has finished $\mathrm{X}$ for its request $\operatorname{Req}(\langle i, s n\rangle, \mathrm{X})$. Therefore, $p_{j}$ cannot attend a different forum while $p_{i}$ is in $\mathrm{X}$. Moreover, given that priorities are unique, when two philosophers request different forums concurrently, the request issued by the low-priority philosopher will be detained by the high-priority philosopher until the high-priority one has finished a forum. So, the two philosophers cannot be in different forums simultaneously. Because neither logically dependent requests nor concurrent requests can violate mutual exclusion, mutual exclusion is guaranteed.

For bounded delay, observe that a philosopher releases all detained messages to its successor when it finishes a forum. Given that a philosopher spends only a finite time in a forum and that every message will eventually be delivered to its target, a philosopher that has issued a request along the ring will eventually receive the request and, so, will eventually attend a forum.

For concurrent entering, observe that philosophers will not detain one another's request if they are interested in the same forum. Given that philosophers not interested in a forum will not detain any request, if some philosophers are interested in the same forum and no philosopher is interested in a different forum, then the philosophers can attend the forum concurrently.

We now consider various complexity measures of CTP-Ring1. It is easy to see that the algorithm requires 
$N$ messages for each philosopher to attend a forum. For time and context-switch complexity, we first calculate the maximum number of passages that may proceed $p_{i}$ 's passage through $\mathrm{X}$ after $p_{i}$ has made a request $\operatorname{Req}\left(\left\langle i, s n_{i}\right\rangle, \mathrm{X}\right)$. Suppose that another philosopher $p_{j}$ has also made a request $\operatorname{Req}\left(\left\langle j, s n_{j}\right\rangle, \mathrm{Y}\right)$. Obviously, if $s n_{j}>s n_{i}$, then $p_{j}$ cannot make a passage through $\mathrm{Y}$ until $p_{i}$ has made a passage through $\mathrm{X}$ (unless $\mathrm{Y}=\mathrm{X}$ ). So assume that $s n_{j} \leq s n_{i}$. Since messages need not be transmitted in the order sent, $p_{j}$ may receive its request while $p_{i}$ 's request is still on the way to, say, $p_{i+1}$. So $p_{j}$ may not have any knowledge about $p_{i}$ 's sequence number $s n_{i}$. Therefore, $p_{j}$ 's next request may still have a sequence number less than or equal to $s n_{i}$ (but greater than $s n_{j}$ ). So $p_{j}$ can still make another passage before $p_{i}$ can attend $\mathrm{X}$. Note that even if the sequence number of the new request is equal to $s n_{i}, p_{j}$ may still attend a forum before $p_{i}$ does if $j<i$. Hence, $p_{j}$ can generate at most $s n_{i}-s n_{j}+1$ passages before $p_{i}$ can attend $\mathrm{X}$. Note that there is no restriction on the type of forums through which the $s n_{i}-s n_{j}+1$ passages are made. So, the $s n_{i}-s n_{j}+1$ passages may constitute $s n_{i}-s n_{j}+1$ rounds.

Moreover, by the way sequence numbers are maintained and that every request message must circulate along the ring once, if there is no pending request then all philosophers have the same sequence number maintained by their $S N$ s. As the maximum $S N$ in the system increases by 1 only if the philosopher holding this number has initiated a new request, in the worst case, the difference between any two $S N$ s cannot exceed $N-1$. To reach this case, some philosopher must have initiated a request, yielding its $S N$ to some number $c$; the request then causes the next philosopher to increase its $S N$ to $c+1$ when initiating another request; the second request then causes the next philosopher to increase its $S N$ to $c+2$ when initiating a third request; and so on. So, in the worst case, when some philosopher $p_{i}$ has initiated a request with sequence number $s n$, the other $N-1$ philosophers may have all made a request, and have the following sequence numbers $s n-(N-1), s n-(N-2), \ldots$, and $s n-1$ maintained by their $S N$ s. If $p_{i}$ 's ID is the smallest, then, by the previous argument, at most $N+(N-1)+\ldots+2=\frac{N^{2}+N-2}{2}$ passages may be initiated before $p_{i}$ can make a passage through a forum. As the $\frac{N^{2}+N-2}{2}$ passages need not overlap or aiming at the same forum, both the time complexity and contextswitch complexity of CTP-Ring 1 are $O\left(N^{2}\right)$.

To measure the algorithm's degree of concurrency, assume that $p_{j}$ is in the meeting room, and $p_{i}$ is waiting for a different forum. Let $s n$ be the sequence number of $p_{i}$ 's request. Similar to above argument, the other $N-2$ philosophers could all have made a request with sequence numbers $s n-(N-2), s n-(N-3), \ldots$, and $s n-1$. Then, at most $(N-1)+(N-2)+\ldots+2=\frac{N^{2}-N-2}{2}$ passages may be initiated before $p_{i}$ can attend its forum. So the degree of concurrency is also $O\left(N^{2}\right)$.

Some simulation results are summarized in Figure 1. In the simulation, we have set up a system of $N$ philosophers and $m$ forums. Each time a philosopher wishes to attend a forum, it randomly chooses one of the $m$ forums to attend, and the choice follows a uniform distribution. The time a philosopher stays in states thinking and talking fol-
$N=30, \mu_{\text {thinking }}=50 \mathrm{~ms}, \mu_{\text {talking }}=250 \mathrm{~ms}, \mu_{\text {link-delay }}=2 \mathrm{~ms}$

\begin{tabular}{|l|c|c|c|c|}
\hline \hline & $m=1$ & $m=3$ & $m=10$ & $m=30^{*}$ \\
\hline average waiting time (ms) & 20.12 & 6108.46 & 7292.95 & 7873.7 \\
\hline average context switches & 0 & 19.08 & 25.91 & 28.86 \\
\hline average round size & NA & 1.51 & 1.11 & 1 \\
\hline average capacity & 30 & 1.49 & 1.11 & 1 \\
\hline average messages per entry & 30 & 30 & 30 & 30 \\
\hline average throughput (entry/sec) & 92.13 & 4.66 & 3.95 & 3.67 \\
\hline
\end{tabular}

Simulation results for Algorithm CTP-Ring1.

\begin{tabular}{|l|c|c|c|c|}
\multicolumn{1}{|c|}{$N=30, \mu_{\text {thinking }}=50 \mathrm{~ms}, \mu_{\text {talking }}=250 \mathrm{~ms}, \mu_{\text {link-delay }}=2 \mathrm{~ms}$} \\
\hline \hline & $m=1$ & $m=3$ & $m=10$ & $m=30^{*}$ \\
\hline average waiting time (ms) & 84.92 & 1538.9 & 3227.53 & 7899.85 \\
\hline average context switches & 0 & 1.93 & 5.34 & 28.87 \\
\hline average round size & NA & 14.64 & 5.37 & 1 \\
\hline average capacity & 30 & 13.11 & 5.06 & 1 \\
\hline average messages per entry & 60 & 60 & 60 & 60 \\
\hline average throughput (entry/sec) & 76.66 & 16.06 & 8.46 & 3.65 \\
\hline
\end{tabular}

Simulation results for Algorithm CTP-Ring2.

\section{Figure 1. Some simulation results}

lows an exponential distribution with means $\mu_{\text {thinking }}$ and $\mu_{\text {talking }}$ respectively. The message transmission time also follows an exponential distribution with a mean $\mu_{\text {link_delay }}$. For comparison, we have also measured the behavior of the algorithm when philosophers use the meeting room in a mutually exclusive style. This is done by designating one unique forum to each philosopher. In the figure, we use $m=N^{*}$ to denote this scenario. We have also set up the case $m=1$ where maximum concurrency should be allowed as no two requests will ever conflict. The average round size measures the average number of passages per round; the average capacity measures, in average, the maximum number of philosophers that can be in the meeting room simultaneously per round. It is not difficult to see that the settings in the figure represent a very high contention situation to the meeting room. From the tables we can see that CTP-Ring 1 provides virtually no concurrency. For example, when $m=3$, it is likely that one third of the pending requests are targeting at the same forum very often. However, the simulation results indicate that the behavior of the system is only slightly better than the case $m=30^{*}$ where the philosophers use the meeting room in a mutually exclusive style! ${ }^{2}$

To see why CTP-Ring1 has such a poor performance, observe that philosophers' requests are granted according to their priorities: a high-priority philosopher must enter the meeting room before a low-priority one. As a result, suppose that a number of philosophers are interested in two different forums (say, $\mathrm{X}$ and $\mathrm{Y}$ ), and their priorities are ordered in such a way that two consecutive philosophers in the ordering are interested in different forums (that is, the first philosopher is interested in $X$, the second philosopher is interested in $\mathrm{Y}$, the third philosopher is interested in $\mathrm{X}$, the fourth philosopher is interested in $Y$, and so on.) Then, the philosophers would proceed their forums in a purely interleaving and sequential style: the first philosopher estab-

\footnotetext{
${ }^{2}$ Some Java applets animating the algorithms for the Congenial Talking Philosophers problem presented in the paper can be found in http://joung.im.ntu.edu.tw/congenial/.
} 
lishes forum $X$, then the second philosopher establishes fo$\operatorname{rum} Y$, then the third philosopher establishes forum $X$, then the fourth philosopher establishes forum $Y$, and so on. Note that, for this case no concurrency is allowed by the algorithm because only one philosopher can be in a forum at a time, albeit there are several other philosophers interested in the same forum. In the following section we present a modification of the algorithm that requires $2 N$ messages per request, but offers a much better performance.

\section{An Improved Algorithm}

As discussed above, the low concurrency of CTP-Ring1 is due to the fact that, if two philosophers $p_{i}$ and $p_{j}$ are interested in the same forum but there is a third philosopher $p_{k}$ interested in a different forum and has a priority in between $p_{i}$ and $p_{j}$, then $p_{i}$ and $p_{j}$ cannot attend the same forum concurrently because the low-priority philosopher $p_{j}$ must wait for $p_{k}$ to finish a forum before it can attend a forum. Our solution for the problem is to let $p_{i}$ issue another message along the ring to "capture" $p_{j}$ (and other philosophers that are also interested in the same forum) so that the captured philosophers can attend the forum concurrently with the captor.

To do so, we introduce a new state checking for the philosophers. As before, a philosopher's priority is set to $\langle i, s n\rangle$ when it issues a request $\operatorname{Req}(\langle i, s n\rangle, \mathrm{X})$. However, the priority may be "upgraded" (but at most once) before the philosopher attends forum $X$. The priority is reset to a minimal value $\langle i, \infty\rangle$ when the philosopher returns to state thinking. (Thus, a philosopher carries a priority all the time. In particular, its priority in state thinking is always lower than that of a philosopher interested in a forum.)

Like the previous algorithm, when a philosopher $p_{i}$ wishes to attend a forum $\mathrm{X}$, it enters state waiting, increments its $S N$ by 1 , and sends a request message $\operatorname{Req}(\langle i, s n\rangle, \mathrm{X})$ to its successor $p_{i+1}$, where $s n$ is the new value of $p_{i}$ 's $S N$. Another philosopher $p_{j}$ in the ring, upon receiving the request, forwards the request if it is also interested in $\mathrm{X}$ or has a priority lower than $\langle i, s n\rangle$; otherwise, $p_{j}$ detains the request in its message queue until it has finished a forum. When the request is returned to $p_{i}, p_{i}$ enters state checking to issue a confirmation message of the form Confirm_ $C(\langle i, s n\rangle, \mathrm{X})$ to its successor $p_{i+1}$. The purpose of the message is twofold: to capture philosophers that are waiting for $\mathrm{X}$, and to make sure that no philosopher is still in a different forum. Philosopher $p_{i}$ must wait until the message is returned before it can attend $X$.

When a philosopher $p_{j}$ receives Confirm_ $C(\langle i, s n\rangle, \mathrm{X})$, where $j \neq i$, it processes the message similarly as request messages: $p_{j}$ forwards the request if it is also interested in $\mathrm{X}$ or has a priority lower than $\langle i, s n\rangle$; otherwise, $p_{j}$ detains the request. Note that all messages (requests and confirmations) detained by a philosopher are released when the philosopher has finished a forum. In addition, if $p_{j}$ is also interested in $\mathrm{X}$, is in state waiting, and has a priority lower than $\langle i, s n\rangle$, then $p_{j}$ is captured by $p_{i}$. In this case, $p_{j}$ assumes $p_{i}$ 's priority and also enters state checking to issue a confirmation message of the form $\operatorname{Confirm}(j,\langle i, s n\rangle, \mathrm{X})$ along the ring. $\left(p_{j}\right.$ 's request message circulating somewhere in the ring then becomes obsolete.) Unlike $p_{i}$ 's confirmation message, $p_{j}$ 's confirmation message serves only one purpose: to make sure that no philosopher is still in a different forum. Therefore, a philosopher receiving $p_{j}$ 's confirmation message processes the message similarly to the other form of confirmation messages (i.e., Confirm_C, where "_C" means "with capturing"), except that the new form of confirmation messages cannot capture the receiving philosophers. This prevents $p_{j}$ from capturing $p_{i}$ after $p_{i}$ has attended $\mathrm{X}$ and has made a new request to re-attend $X$, thereby preventing $p_{i}$ and $p_{j}$ from capturing each other repeatedly (which could also result in a violation of mutual exclusion).

Philosopher $p_{i}$ enters state talking to attend $\mathrm{X}$ when its confirmation message Confirm_ $C(\langle i, s n\rangle, \mathrm{X})$ is returned. After $p_{i}$ has finished the forum, it forwards all the messages it has detained to its successor $p_{i+1}$ and then returns to state thinking. Note that because some low-priority philosophers may have been captured by $p_{i}$ to concurrently attend $\mathrm{X}$, after $p_{i}$ has exited $\mathrm{X}$, some philosophers may still be in $\mathrm{X}$ while another philosopher $p_{k}$ whose priority is higher than the captured philosophers is waiting for a different forum $\mathrm{Y}$. Moreover, because $p_{i}$ will release all messages held in its message queue when it exits $\mathrm{X}, p_{k}$ may have received its own request $\operatorname{Req}\left(\left\langle k, s n^{\prime}\right\rangle, \mathrm{Y}\right)$ after $p_{i}$ has exited $\mathrm{X}$ but before all the philosophers captured by $p_{i}$ have exited $\mathrm{X}$. Therefore, $p_{k}$ cannot immediately enter state talking to attend $Y$ when it receives its own request. Instead, it must initiate a confirmation message Confirm_ $C\left(\left\langle k, s n^{\prime}\right\rangle, \mathrm{Y}\right)$ to make sure that all $p_{i}$ 's captured philosophers have exited $\mathrm{X}$. Similarly, any philosopher captured by $p_{k}$ during the time $p_{k}$ 's confirmation message is circulating must also initiate a confirmation message (of the form Confirm) along the ring before it can enter state talking to attend a forum.

We refer to the new algorithm as CTP-Ring2, and its complete code is given in Figure 2. The algorithm is represented by a CSP-like repetitive command of the form [1]:

$$
*\left[g_{1} \rightarrow s_{1} \square g_{2} \rightarrow s_{2} \square \ldots \square g_{k} \rightarrow s_{k}\right]
$$

The guarded command $s_{i}$ is executed only if its guard $g_{i}$ is enabled: The Boolean expression (if any) evaluates to true and the message specified in the guard (if any) has arrived. If more than one guarded command have an enabled guard, then one of them is chosen for execution, and the choice is nondeterministic. We assume that a guarded command whose guard is continuously enabled will eventually be executed.

It is not difficult to see that CTP-Ring2 satisfies mutual exclusion, bounded delay, and concurrent entering. Due to the space limitation, the correctness of the algorithm is proved in the full paper [6]. The algorithm has similar complexity measures as CTP-Ring1; that is, its time complexity, context-switch complexity, and degree of concurrency are all of $O\left(N^{2}\right)$. Again, the analysis can be found in the full paper. We remark here that, by a simple modification, the context-switch complexity of CTP-Ring2 can be further reduced to $\min (N, m+1)$. To do so, request messages now have this form:

- $\operatorname{Req}\left(\langle i, s n\rangle, s n_{\max }, \mathrm{X}\right)$

where the extra sequence number $s n_{\max }$ carried by the request is the maximum sequence number of the philosophers through which the request has passed. Initially, $s n_{\max }$ 
*[wish to attend a forum of $\mathrm{X} \longrightarrow$

$S N:=S N+1$

target $:=\mathrm{X}$;

priority $:=\langle i, S N\rangle$;

state $:=$ waiting;

send $\operatorname{Req}(\langle i, S N\rangle, \mathrm{X})$ to $p_{i+1}$;

$\square$ receive $\operatorname{Req}(\langle j, s n\rangle, \mathrm{Y}) \longrightarrow$

$S N:=\max (S N, s n)$;

$[i=j \wedge$ state $=$ waiting $\wedge$ priority $=\langle j, s n\rangle \longrightarrow$ state $:=$ checking;

send Confirm_ $C(\langle i, s n\rangle$, target $)$ to $p_{i+1}$

$\square i \neq j \wedge($ target $=\mathrm{Y} \bigvee($ priority $\langle\langle j, s n\rangle) \longrightarrow$ send $\operatorname{Req}(\langle j, s n\rangle, \mathrm{Y})$ to $p_{i+1}$;

$\square i \neq j \wedge$ target $\neq \mathrm{Y} \wedge$ priority $\rangle\langle j$, sn $\rangle \longrightarrow$ add $\operatorname{Req}(\langle j, s n\rangle, \mathrm{Y})$ to message_queue;

$\square$ else $\longrightarrow$ skip; $/ * p_{i}$ receives its own obsolete request $\left.* /\right]$

$\square$ receive Confirm_ $C(\langle j, s n\rangle, Y) \longrightarrow$

[ $i=j \longrightarrow /$ receive $p_{i}$ 's own confirmation message */ state $:=$ talking; attend a forum of target;

$\square i \neq j \wedge$ target $\neq \mathrm{Y} \wedge$ priority $>\langle j$, sn $\rangle \longrightarrow$ add Confirm_ $C(\langle j, s n\rangle, \mathrm{Y})$ to message_queue;

$\square$ else $\longrightarrow$ send Confirm_C $(\langle j, s n\rangle, Y)$ to $p_{i+1}$; [ target $=\mathrm{Y} \wedge$ state $=$ waiting

$$
\bigwedge \text { priority }<\langle j, s n\rangle \longrightarrow
$$

state $:=$ checking $; / *$ captured by $p_{j} * /$

priority $:=\langle j, s n\rangle ;\left.\right|^{*}$ assume the captor's priority $* /$

send $\operatorname{Confirm}(i,\langle j, s n\rangle$, target $)$ to $\left.\left.p_{i+1} ;\right]\right]$

$\square$ receive $\operatorname{Confirm}(j,\langle k, s n\rangle, Y) \longrightarrow$

[ $i=j \longrightarrow / *$ receive $p_{i}$ 's own confirmation message $* /$ state $:=$ talking;

attend a forum of target;

$\square i \neq j \wedge$ target $\neq \mathrm{Y} \wedge$ priority $>\langle k, s n\rangle \longrightarrow$ add $\operatorname{Confirm}(j,\langle k, s n\rangle, \mathrm{Y})$ to message_queue;

$\square$ else $\longrightarrow$ send $\operatorname{Confirm}(j,\langle k, s n\rangle, \mathrm{Y})$ to $\left.p_{i+1} ;\right]$

$\square$ exit a forum of target $\longrightarrow$

state $:=$ thinking;

target $:=\perp$;

priority $:=\langle i, \infty\rangle ; / *$ reset the priority to a minimal value $* /$

for each $m s g \in$ message_queue do send $m s g$ to $p_{i+1}$;

message_queue $:=\emptyset$;

41

\section{variables \& messages:}

- state: the state of $p_{i}$. Its value ranges over $\{$ thinking, waiting, checking, talking , and is initialized to thinking.

- $S N$ : the sequence number maintained by $p_{i}$. It is initialized to 0 .

- target: the forum $p_{i}$ wishes to attend, or $\perp$ otherwise. It is initialized to $\perp$.

- priority: $p_{i}$ 's priority. It is initialized to a minimal value $\langle i, \infty\rangle$.

- message_queue: the queue of messages detained by $p_{i}$. It is initialized to empty.

- $R e q(\langle i, s n\rangle, \mathrm{X})$ : a request message by $p_{i}$ to attend a forum $\mathrm{X}$, where $\langle i, s n\rangle$ is $p_{i}$ 's priority when it makes the request.

- Confirm_C $(\langle i, s n\rangle, \mathrm{X})$ : a confirmation message issued by $p_{i}$ (with priority $\langle i, s n\rangle$ ) to capture philosophers and to make sure that no philosopher is still in a different forum.

- Confirm $(i,\langle j, s n\rangle, \mathrm{X})$ : a confirmation message issued by $p_{i}$ to make sure that no philosopher is still in a different forum. $\langle j, s n\rangle$ is the priority of $p_{i}$ assumed from its captor $p_{j}$.

Figure 2. Algorithm CTP-Ring2 for $p_{i}$. equals to $p_{i}$ 's $S N$ when $p_{i}$ issues the request (and note that $s n$ is also equal to $p_{i}$ 's $S N$ ). Upon receiving the request, a philosopher $p_{j}$ adjusts its own $S N$ to be maximum value of its current $S N$ and $s n_{\max }$. When $p_{j}$ is to forward the request to its successor (in the case that $i \neq j$ ), it adjusts $s n_{\max }$ to be maximum value of its current $S N$ and $s n_{\max }$. (Note that if the request is to be held by $p_{j}$, then in between the time $p_{j}$ receives the request and the time it releases the request, $p_{j}$ 's sequence number $S N$ may have been adjusted a number of times.)

An extra sequence number is also added to confirmation messages so that they now have one of the following two forms:

- Confirm_C $\left(\langle i, s n\rangle, s n_{\max }, \mathrm{X}\right)$

- $\operatorname{Confirm}\left(i,\langle j, s n\rangle, s n_{\max }, \mathrm{X}\right)$.

The extra parameter $s n_{\max }$ is initialized and used as in the new request messages.

The simulation results of the new algorithm are also given in Figure 1. From the results we can see that the new algorithm significantly improves the performance of CTP-Ring1.

\section{Conclusions}

We have presented two distributed algorithms CTP-Ring1 and CTP-Ring2 for the Congenial Talking Philosophers problem on a ring network where each philosopher can only communicate directly with its two neighboring philosophers. CTP-Ring1 requires $N$ messages per entry to the meeting room, and the time complexity, context-switch complexity, and degree of concurrency are all $O\left(N^{2}\right)$, where $N$ is the total number of philosophers in the ring. In contrast, CTP-Ring 2 requires $2 N$ messages, has also $O\left(N^{2}\right)$ time complexity and degree of concurrency, but has a better context-switch complexity of $\min (N, m+1)$, where $m$ is the total number of forums the philosophers may attend. Although, statically, the two algorithms do not differ very much from various complexity measures, the difference between their dynamic performance is significant. As we have shown in the simulation, CTP-Ring1 performs poorly as compared to CTP-Ring2; it out-performs CTP-Ring2 only in message complexity.

\section{References}

[1] C. A. R. Hoare. Communicating sequential processes. $C A C M$, 21(8):666-677, Aug. 1978.

[2] Y.-J. Joung. Asynchronous group mutual exclusion (extended abstract). In Proc. 17th ACM PODC, pp. 51-60, 1998.

[3] Y.-J. Joung. The congenial talking philosophers problem in computer networks. Technical report, Dept. of Info. Management, National Taiwan University, Taipei, Taiwan, 1998.

[4] L. Lamport. Time, clocks and the ordering of events in a distributed system. CACM, 21(7):558-565, July 1978.

[5] A. M. Law and W. D. Kelton. Simulation modeling \& analysis. McGraw-Hill, 1991.

[6] K.-P. Wu and Y.-J. Joung. Asynchronous group mutual exclusion in ring networks. Technical report, Dept. of Info. Management, National Taiwan University, Taipei, Taiwan, 1999. 